\title{
Hand Writing Board - Online Tool Used as a Means of Interactive Communication Using Images
}

\author{
Krunal Pande ${ }^{1}$, Kunal Khadkeshwar' ${ }^{2}$ Nachiket Satpute ${ }^{3}$, \\ Pranshu Singh ${ }^{4}$, Yash Gupta ${ }^{5}$ and Vrushali Bongirwar ${ }^{6}$ \\ 1, 2, 3, 4, 5, 6 Shri Ramdeobaba College of Engineering and \\ Management, Nagpur, Maharashtra, India
}

\section{ABSTRACT}

This is a web application which can be used for two way communication. In the world of the pandemic where the face to face meeting and classes are affected this application can be used to communicate in this situation. The application provides a feature where one member has to create a room and then share the link of the room with other member. Both video and text chat option are available in the application. During the meeting or class members can in real time share the video of the sheet where one is writing or drawing. Also one can take a picture of the sheet and the picture is shared with all member. For the creation of room and video and text chat WebRTC module was used as it provides a real time communication ability and in fast. When we click a photo we need to erase the background, for this we used binarization. Using the binarization technique we eliminated the white background of the picture so that only the written or drawn part is visible. One the image is binarized it is send to the members. Then anyone who wants to append his/ her side in the same image can do so which is implemented by image overlay method. Thus the member's interaction can be a two way communication.

\section{KEY WORDS: WEBRTC, BINARIZATION, IMAGE OVERLAY, ONLINE COMMUNICATION}

\section{INTRODUCTION}

There are challenges in taking classes online or holding an online meeting under the influence of corona. So, video meet in a good method to communicate [Sullivan, 2012]. In face-to-face class and at meetings, one can see the process of having someone write on a white board, handwriting on the board, and having another student or co-worker take notes. Now, there are a lot of scenes where it is very disruptive to not be able to do this, and

\section{ARTICLE INFORMATION}

*Corresponding Author: pandekv@rknec.edu

Received 15th Oct 2020 Accepted after revision 25th Dec 2020

Print ISSN: 0974-6455 Online ISSN: 2321-4007 CODEN: BBRCBA

Thomson Reuters ISI Web of Science Clarivate Analytics USA and Crossref Indexed Journal

\section{Clarivate
Analytics}

NAAS Journal Score 2020 (4.31)

A Society of Science and Nature Publication,

Bhopal India 2020. All rights reserved.

Online Contents Available at: http//www.bbrc.in/

Doi: http://dx.doi.org/10.21786/bbrc/13.14/34 they often communicate in one way and it is difficult to say that it is in a normal place.

We read different papers for a way of communication and also found their limitations [Merchant J., 2016][ Mandy M., Rachel C., Mavourneen G. Caseyand M 2009] [ Chaimeeboonand J., Namee K 2017].After reading and discussion we came to a certain conclusion. All you need is a white paper, a black pen and a smartphone. Members in one room can see what they are writing in real time and edit it together. Using this application one can track the process in real time to find out which student process. Teachers are far away and can find where they stumble, making the answer easier. Classes and meetings such as going around the white board with several people will be able to be done online.

Literature Review: Md. Habibur Rahaman has surved on the real-time and a fast solution for communication which helped our thinking[Rahaman M. 2015]. The importance of video chat/conference can be taken in note 
from the paper of Sullivan[Sullivan, Jessica R. 2012]. From the papers of Jirayu Chaimee boonand Khanista Namee [Chaimeeboonand J., Namee K 2017] about Cisco Webx, John Merchant[Merchant J., 2016] about skype,Mandy M. Archibald1, Rachel C. Ambagtsheer, Mavourneen G. Caseyand Michael Lawless[Mandy M., Rachel C., Mavourneen G. Caseyand M 2009] about zoom, we found out that there is no method of communication as of ours which uses images for two way interaction. After going through this paper we came up with the idea of sharing images and able to communicate with the members along with voice and text chat.

Zinah Tareq Nayyef, Sarah Faris Amer [Zinah N, Sarah F 2018] paper and also paper of Cui Jian, Zhuying Lin [Jian C., Lin Z 2015] shows the implementation and importance of peer to peer communication using WebRtc. From the paper of Wan Azani Mustafa, Mohamed Mydin M. Abdul Kader [Wan M. 2018] we got to know about the binarization of image. For the threshold value of binarization Wan Azani[Wan M. 2018] Mustafa proposed the value. Paper of J. Trein, A. Th. Schwarzbacher, B. Hoppe, K.-H. Noffzand T. Trenschel[J. Trein, As Th., Hoppe B., Noffzand T 2007.] helped us with the idea of image overlapping. Christina Davidson's[Davidson C. 2009] paper gave us a thought to think about transcription and so does the paper of Karez Abdul wahhab Hamad , Mehmet Kaya [Karez H. ,Kaya H. 2016] about Optical Character Recognition .

\section{MATERIAL AND METHODS}

App Flow

1. Create a room.

2. Share the link made to students / colleagues.

3. After the meeting has started one can send the necessary pictures to the room.

4. Click on the image

5. Binarize the snapshot.

6. Send a photo to all users in the same room.

7. Change the white part of the bulk image and display the camera image below the scanned image.

8. This process can be repeated several times.

9. Send photos from your camera to users in the same room.

10. You can now control which photos are displayed on your camera or in a third party camera.

3.1. WebRtc: Significant advances in present day innovation in sending sight and sound and PC interchanges require elevated level correspondence between individuals [Zinah N, Sarah F 2018]. To meet this developing need, a continuous correspondence measure has been created. Continuous (RTC) correspondence is another and extensive approach to grow a web perusing model that permits admittance to data, for example, web-based media, talk, video, and online TV, just as coordinated interchanges. Clients of these applications can see, record, remark, or transfer video content. In the open source application WebRTC has empowered clients of these applications to see video substance or
Figure 1: WebRTC support in browsers

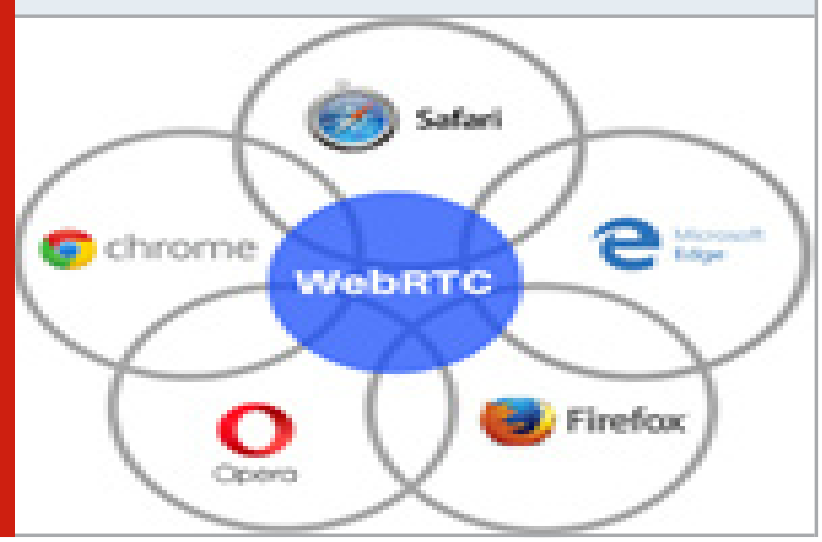

record, remark or stream on it to accomplish ongoing correspondence between internet browsers. .

WebRTC is a type of constant correspondence innovation that has improved API (Application Programming Interface) innovation to empower ongoing mixed media transmission, for example, voice, and video (counting codes) accessible on an internet browser without a top notch sight and sound correspondence module from Peer-to-peer is accessible to web designers without the conventional module highlights utilizing JavaScript codes. WebRTC is an open source accessible to Google in 1122 for mixed media web applications. Presently, it is introduced on Web programs, for example, Firefox, Chrome, drama, and so on. Later on, this innovation will be applied to all programs aside from module parts, which can eliminate viral weaknesses while giving intelligent network. presents a program that pays for sight and sound web-based features, for example, video and video, distinguishes the client and finds different clients of the program, fulfills the essential prerequisites that will be viewed as secure without establishment or complex activity inside an internet browser on different gadgets and applications utilizing WebRTC.

WebRTC follows the semantics-server client promoter in the sense of peer-to-peer communication between browsers. Connectivity controls the media path to allow direct flow between browsers. Network signals are transmitted during Web Server which helps to convert, translate or manage signals, as required by WebSocket or HTTP. It was noted that the interface between the browser and the server is not the same as in WebRTC, where it is part of the application. Web servers can communicate using a standard signature protocol such as SIP (Session Initiation Protocol) or Jingle. Otherwise, the property is signed the law may be used for this purpose microphone, to more advanced video streaming and screen sharing apps.

3.2. Signalling and Negotiation: We need to know where user is located to connect with the user. The IP address allows to the Internet enabled devices to send information/data directly between each other. RTC Peer Connection is used for this. After locating each other on 
the internet, they start to share/exchange information between each other. They share information about which protocols and devices each device supports. Only Thing user need to do is exchange details of contact and rest of the process is done by WebRTC. This process is called as Signalling and Negotiation [Jian C., Lin Z, 2015].

\section{There are some steps mentioned below:}

1. Create a list which contains candidates for peer connection.

2. User needs to choose candidates for peer connection.

3. Another user is notified by Signalling layer about connection request.

4. User who sent request is notified of the status of the offer.

5. First user starts RTC Peer Connection with requested user.

6. They start to exchange the software and hardware information by means of signalling server.

7. They share location information with each other.

8. At last Connection is successfully established or failed.

3.3. Binarization: Binarization means converting a colour image into an image created only with black and white pixels[Mustafa A., Mohamed M. Abdul Kader, 2018]. A binary image contains only of black and white pixels, 0 for white and 1 for black. Used in OCR and Image Processing, and a Grayscale Image has all the pixels in a range 0-255 of grey. We used greyscale images to distinguish between object parameters and paper background.

Figure 2: Comparing frequency v/s pixel value

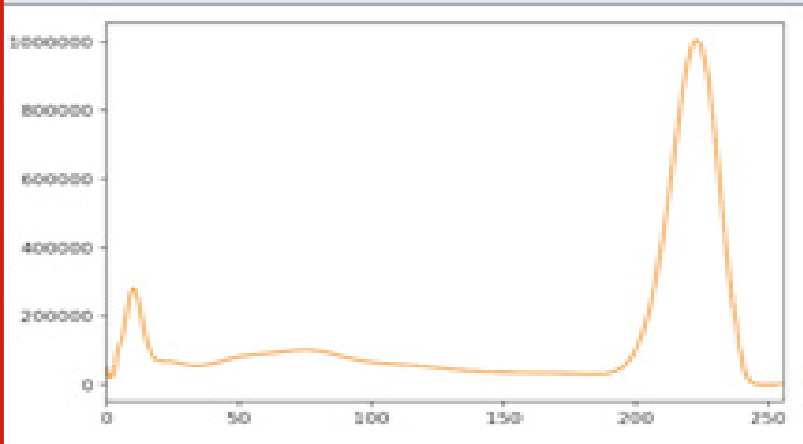

We utilized thresholding to change over grayscale picture to paired picture[Mustafa A., Mohamed M. Abdul Kader, 2018]. It fragments all pixels to either 1 or 0 . All pixels (0-255) are planned and pixels crossing edge are fragmented to 1 or 0,1 being white and 0 being dark. For characterizing the limit an incentive there are different methods. The calculation introduced in this work comprises of two stages. It consolidates the basic parallel thresholding and OTSU thresholding. It computes the t-esteem for which the weighted inside class difference of the two classes, 1 and 0 , are limited.
In the Image, threshold is decided by comparing frequency v/s pixel value. Implementation using OpenCV cv2.threshold method, in JS or NodeJS. Cv2.threshold has options for thresholding as: THRESH_BINARY, THRESH_ BINARY_INV , THRESH_TRUNC , THRESH_TOZERO. Used THRESH_BINARY to convert image pixels above threshold.

\section{Code Snippet:}

img = cv2.imread('gradient.png',0)

ret,thresh1 = cv2.threshold(img, 127,255,cv2.THRESH_ BINARY)

How Will Binarization be used?

- Binarization will convert user captured image to 1 bit image. Now, the white part will be treated as transparent and only the black colour image will be displayed on the camera.

- User will capture a diagram and turn in for other users to modify. The other users will modify the image and turn it in for others to see. The overlap will reduce efforts of redrawing the entire image.

- Binarization will help in detecting transparent part of image since it consists only of black and white pixels, which are either 0 for white or 1 for black

- Implementation: Binarization can be achieved through thresholding technique in OpenCV using JavaScript. ( cv.threshold, cv.adaptiveThreshold etc.)

\section{Figure 3: Image Overlay}

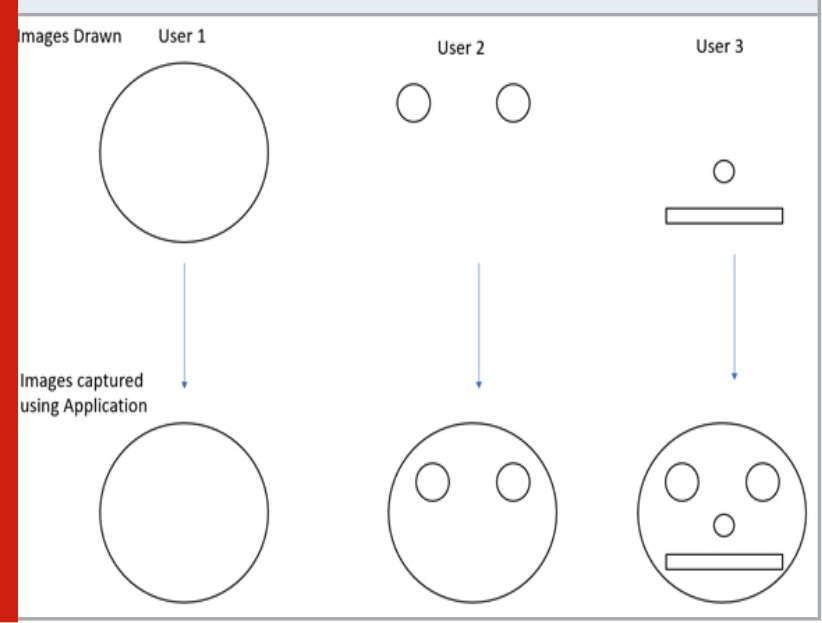

3.4. Image Overlay: Image overlay is used to place captured images by the user onto the already present image[J. Trein, As Th., Hoppe B., Noffzand T, 2007]. This is achieved using HTML and CSS. First place the background image as relative so Next place the overlay image as relative to the first image. Then images are formatted. Z-index is used to decide stack ordering of image.

\section{RESULTS AND DISCUSSION}

There are many of instruments for online communication that can be seen together in the world, the most significant 
thing was to have the option to share manually written things progressively and alter them on the spot, so we zeroed in on that point. We were also stuck on running on a browser without installing an app so that anyone could easily achieve this. Ability to chat the members. Video Call facility for all to access. In the future we can also add the transcription and Optical Character Recognition (OCR) in the application. We thought of this topic and had a brief discussion about it. It will be helpful for the users. Transcription is a method of converting audio to text [Christina Davidson, 2009]. OCR is a technique to process the image and gather all text in the image [Karez H. ,Kaya H., 2016].

\section{CONCLUSION}

Rather than single direction correspondence like onrequest conveyance, this application can without much of a stretch and very much impart progressively with any individual who has white paper, dark pen, and cell phone.

\section{REFERENCES}

Christina Davidson. 2009. "Transcription: Imperatives for Qualitative Research”, Int. J. Qual. Methods 8, 2 (2009), pp. 35-52.

Cui Jian, Zhuying Lin, Beijing, "Research and Implementation of WebRTC Signaling via WebSocketbased for Real-time Multimedia Communications", 5th International Conference on Computer Sciences and Automation Engineering, ICCSAE 2015, pp. 374-380 J. Trein, A. Th. Schwarzbacher, B. Hoppe, K.-H. Noffzand T. Trenschel, "Development of a FPGA Based Real-Time Blob Analysis Circuit”, ISSC 2007, Derry N. Ireland, 2007, pp. 121-126.

Jirayu Chaimeeboonand Khanista Namee, "Implementation a WebEx Conferencing Testbed for DLIT:Classroom”, the 9th International Conference on Sciences, Technology and Innovation for Sustainable
Well-Being (STISWB 2017) June 2017, pp.1-9 John Merchant, Sydney,2016 "The use of Skype in analysis and training: a research and literature review", Journal of Analytical Psychology, Volume 61, Issue 3, pp.309-328

Karez Abdulwahhab Hamad, Mehmet Kaya 3rd September 2016. "A Detailed Analysis of Optical Character Recognition Technology”, International Journal of Applied Mathematics, Electronics and Computers Advanced Technology and Science IJAMEC, 2016, 4(Special Issue), pp. 244-249

Mandy M. Archibald1, Rachel C. Ambagtsheer, Mavourneen G. Caseyand Michael Lawless, "Using Zoom Video conferencing for Qualitative Data Collection: Perceptions and Experiences of Researchers and Participants International Journal of Qualitative Methods.” ,Volume 18, 13.09.2019 , pp.1-8

Md. Habibur Rahaman, "A Survey on Real-Time Communication for Web”, Scientific Research Journal (SCIRJ, Issue VII, ), Volume III, Issue VII, July 2015, pp.39-44

Sullivan, "The potential for video conferencing as a research tool is almost unlimited" ,2012, pp. 60

Wan Azani Mustafa, Mohamed Mydin M. Abdul Kader, "Binarization of Document Images: A Comprehensive Review”, 1st International Conference on Green and Sustainable Computing (ICoGeS) 2017,IOP Conf. Series: Journal of Physics: Conf. Series 1019012023 , Volume 1019, 25-27 November 2017, pp. 1-9

Wan Azani Mustafa1,"A proposed optimum threshold level for document image binarization”,Journal of Advanced Research in Computing and Applications Volume 7, Issue 1, 2017, pp.8-14

Zinah Tareq Nayyef, Sarah Faris Amer, "Peer to Peer Multimedia Real-TimeCommunication System based on WebRTC Technology”, International Journal of Engineering \& Technology, 7 (2.9), 2018, pp.125-130. 